Rayleigh Scattering Diagnostic for Measurement of Temperature and Velocity in Harsh Environments

Richard G. Seasholtz and Lawrence C. Greer III

Lewis Research Center, Cleveland, Ohio 
Since its founding, NASA has been dedicated to the advancement of aeronautics and space science. The NASA Scientific and Technical Information (STI) Program Office plays a key part in helping NASA maintain this important role.

The NASA STI Program Office is operated by Langley Research Center, the Lead Center for NASA's scientific and technical information. The NASA STI Program Office provides access to the NASA STI Database, the largest collection of aeronautical and space science STI in the world. The Program Office is also NASA's institutional mechanism for disseminating the results of its research and development activities. These results are published by NASA in the NASA STI Report Series, which includes the following report types:

- TECHNICAL PUBLICATION. Reports of completed research or a major significant phase of research that present the results of NASA programs and include extensive data or theoretical analysis. Includes compilations of significant scientific and technical data and information deemed to be of continuing reference value. NASA's counterpart of peerreviewed formal professional papers but has less stringent limitations on manuscript length and extent of graphic presentations.

- TECHNICAL MEMORANDUM. Scientific and technical findings that are preliminary or of specialized interest, e.g., quick release reports, working papers, and bibliographies that contain minimal annotation. Does not contain extensive analysis.

- CONTRACTOR REPORT. Scientific and technical findings by NASA-sponsored contractors and grantees.
- CONFERENCE PUBLICATION. Collected papers from scientific and technical conferences, symposia, seminars, or other meetings sponsored or cosponsored by NASA.

- SPECIAL PUBLICATION. Scientific, technical, or historical information from NASA programs, projects, and missions, often concerned with subjects having substantial public interest.

- TECHNICAL TRANSLATION. Englishlanguage translations of foreign scientific and technical material pertinent to NASA's mission.

Specialized services that complement the STI Program Office's diverse offerings include creating custom thesauri, building customized data bases, organizing and publishing research results ... even providing videos.

For more information about the NASA STI Program Office, see the following:

- Access the NASA STI Program Home Page at http://www.sti.nasa.gov

- E-mail your question via the Internet to help@sti.nasa.gov

- Fax your question to the NASA Access Help Desk at (301) 621-0134

- Telephone the NASA Access Help Desk at (301) 621-0390

- Write to:

NASA Access Help Desk

NASA Center for AeroSpace Information 7121 Standard Drive

Hanover, MD 21076 
NASA/TM-1998-206980

AIAA-98-0206

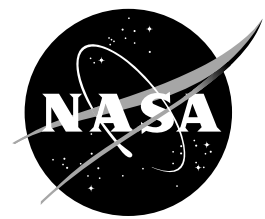

Rayleigh Scattering Diagnostic for Measurement of Temperature and Velocity in Harsh Environments

Richard G. Seasholtz and Lawrence C. Greer III

Lewis Research Center, Cleveland, Ohio

Prepared for the

36th Aerospace Sciences Meeting and Exhibit

sponsored by the American Institute of Aeronautics and Astronautics

Reno, Nevada, January 12-15, 1998

National Aeronautics and

Space Administration

Lewis Research Center 


\section{Acknowledgments}

We would like to acknowledge the diligent efforts of Mr. W. Trevor John, who was responsible for setting up and aligning the optical system used for this work. Also, we thank Prof. G. Tenti for providing us with the computer code for his 6 moment Rayleigh scattering model.

Available from

NASA Center for Aerospace Information 7121 Standard Drive

Hanover, MD 21076

Price Code: A03
National Technical Information Service 5287 Port Royal Road Springfield, VA 22100 Price Code: A03 


\title{
Rayleigh Scattering Diagnostic for Measurement of Temperature and Velocity in Harsh Environments
}

\author{
Richard G. Seasholtz ${ }^{*}$ and Lawrence C. Greer III ${ }^{\dagger}$ \\ NASA Lewis Research Center \\ Cleveland, $\mathrm{OH} 44135$
}

\begin{abstract}
A molecular Rayleigh scattering system for temperature and velocity measurements in unseeded flows is described. The system is capable of making measurements in the harsh environments commonly found in aerospace test facilities, which may have high acoustic sound levels, varying temperatures, and high vibration levels. Light from an argon-ion laser is transmitted via an optical fiber to a remote location where two flow experiments were located. One was a subsonic free air jet; the second was a low-speed heated air jet. Rayleigh scattered light from the probe volume was transmitted through another optical fiber from the remote location to a controlled environment where a Fabry-Perot interferometer and cooled CCD camera were used to analyze the Rayleigh scattered light. Good agreement between the measured velocity and the velocity calculated from isentropic flow relations was demonstrated $(<5 \mathrm{~m} / \mathrm{sec})$. The temperature measurements, however, exhibited systematic errors on the order of $10-15 \%$.
\end{abstract}

\section{Introduction}

Molecular Rayleigh scattering offers a means to measure gas flow parameters including density, temperature, and velocity. The Rayleigh scattered power is proportional to the gas density, the spectral width is related to the gas temperature, and the shift in the frequency of the spectral peak is proportional to one component of the bulk velocity. Since Rayleigh scattering is a molecular scattering process, no seeding of the flow is necessary. It thus finds application in measurement situations where it is not possible to seed the flow, or where seeding is not feasible because of contamination concerns. The technique has been successfully used in a variety of applications including free jets ${ }^{1,2,3}$, rocket exhaust plumes ${ }^{4}$, and wind tunnels ${ }^{5,6}$.

Many applications of Rayleigh scattering diagnostics are in test facilities, where the environment near the test section frequently has high acoustic noise levels, mechanical vibrations, and varying temperatures. This environment is not conducive to the stable operation of some of the equipment used for measuring

\footnotetext{
${ }^{*}$ Senior Research Engineer, Member AIAA

${ }^{\dagger}$ Electronics Engineer
}

the Rayleigh scattering spectrum. In particular, the Fabry-Perot interferometer is very sensitive to vibration and temperature changes. One way to avoid this problem is to locate the delicate optical equipment in a remote location having a more benign environment. For example, in a previous test program in the Lewis $9 \times 15$ foot subsonic wind tunnel ${ }^{6}$, the laser $(\mathrm{CW}$ argonion) was located about 200 meters from the test section and the laser light was transmitted through an optical fiber. The Rayleigh scattered light was transmitted through optical fiber to a separate room, where a scanning Fabry-Perot interferometer was used to measure the Rayleigh scattering spectrum. Use of the Fabry-Perot in the scanning mode means that only time average measurements can be obtained. Other Rayleigh scattering diagnostics use the Fabry-Perot in a static imaging mode ${ }^{1-3}$, where the Rayleigh scattering spectrum can be obtained with a single laser pulse. However, this technique required that the Fabry-Perot and image detector be located close to the measurement region. Operation in high-noise environments requires use of an acoustic enclosure.

In this paper, we describe a new technique where the light from the measurement region is transmitted through an optical fiber to a remote location. Light exiting the fiber is imaged through a static Fabry-Perot interferometer onto an image detector, in a manner similar to the previously developed planar Rayleigh scattering systems. However, because the light is transmitted through the single optical fiber, the one-toone imaging characteristic is lost. The described system thus measures the flow parameters at a point. No scanning of the interferometer is used, which means that the measurement time is determined only by the time needed to detect sufficient photons to give the desired measurement accuracy. The source laser can be either a CW laser or a pulsed laser. Use of a single laser pulse will give instantaneous measurements. If a $\mathrm{CW}$ laser is used, the laser light can be transmitted to the test section through an optical fiber. Unfortunately, the damage threshold of currently available optical fibers is less than the pulse energy $(\sim 1 \mathrm{~J})$ of Q-switched lasers commonly used for Rayleigh scattering diagnostics? This means that either conventional optics, or fiber optics bundles, must be used to transmit the light from pulsed lasers to the test region. 
In addition to providing a means to make measurements in harsh environments, this technique should result in more accurate measurements than those previously obtained using a light sheet because an entire circular fringe is used. This means any shift of the center of the fringe will not result in errors in temperature and velocity. This problem is suspected as the cause of errors in previous work ${ }^{3}$ in a free jet where the velocities obtained from opposite sides of a fringe were symmetrically shifted from the actual velocity. Use of optical fibers also simplifies the simultaneous measurement of two or three velocity components.

\section{Theory}

\section{Molecular Rayleigh scattering}

Rayleigh scattering ${ }^{8}$ is a result of interaction of an electric field with an atom or molecule. Because the wavelength is much larger than the size of a molecule, a dipole moment is induced that oscillates and radiates at the frequency of the incident field. This is an elastic scattering process, where the internal energy of the molecule is not changed, so the frequency of the scattered light is equal to the frequency of the incident light, altered only by the Doppler effect due to the motion of the molecules. Because of the random spatial distribution of the molecules in a gas, the total intensity of the scattered light from a volume of gas is the sum of the intensities of the light scattered from the individual molecules. At low gas densities, molecular interactions are rare and the Rayleigh scattering spectrum is determined only by the molecular velocity distribution; for the usual Maxwellian velocity distribution, the spectrum is Gaussian. However, at higher gas density, the molecular motions become correlated and the character of the spectrum changes. The spectrum can be analyzed either by considering the scattering from the individual molecules with proper accounting of the collective effects or by considering the scattering as being caused by fluctuations in the gas density.

For a gas with number density $n$ illuminated by a laser with wavelength $\lambda$ and energy $E_{o}$, the number of detected photoelectrons is

$$
N_{R}=\frac{\varepsilon E_{o} n L_{x} \lambda \Omega}{h c}\left(\frac{\mathrm{d} \sigma}{\mathrm{d} \Omega}\right) \sin ^{2} \chi
$$

where $L_{x}$ is the length along the beam of the scattering volume, $\Omega$ is the solid collection angle, $\varepsilon$ is the overall collection efficiency (including the detector quantum efficiency), $h$ is Planck's constant, $c$ is the velocity of light, $\mathrm{d} \sigma / \mathrm{d} \Omega$ is the differential scattering cross section, and $\chi$ is the angle between the electric field vector of the (linearly polarized) incident light and the direction of the scattered light. Generally experiments are arranged so the incident electric vector is perpendicular to the scattering plane (i.e., " $s$ " type) and $\chi=90^{\circ}$; for this situation the scattering is independent of the scattering angle. However, if the electric vector is in the scattering plane (i.e., " $p$ " type), the scattering is dependent on the scattering angle and approaches zero at a $90^{\circ}$ scattering angle.

The Rayleigh differential scattering cross section is related to the index of refraction $\mu$ of the gas by

$$
\left(\frac{d \sigma}{d \Omega}\right)=\frac{4 \pi^{2}}{\lambda^{4} n^{2}}(\mu-1)^{2}
$$

where $n$ is the number density of the gas. Published tabulations ${ }^{9,10,11}$ of refractive index data are useful for calculating Rayleigh scattering cross sections.

\section{Rayleigh scattering spectrum}

The Rayleigh scattering spectrum is usually expressed as a function of a non-dimensional frequency $x$ and a non-dimensional collision frequency $y$

$$
x=\frac{2 \pi\left(f-f_{o}\right)}{K a}, \quad y=\frac{p}{\eta K a}
$$

where $f-f_{o}$ is the frequency shift of the scattered light relative to the frequency of the incident light, $p$ is the gas pressure, $\eta$ is the shear viscosity, $K=$ $(4 \pi / \lambda) \sin \left(\theta_{S} / 2\right)$ is the magnitude of the interaction wave vector $\mathbf{K}=\mathbf{k}_{\mathrm{s}}-\mathbf{k}_{\mathbf{0}}$ (with $\mathbf{k}_{\mathbf{0}}$ and $\mathbf{k}_{\mathrm{s}}$ being the wave vectors of the incident and scattered light), $\theta_{\mathrm{s}}$ is the scattering angle, and $a=(2 \kappa T / m)^{1 / 2}$ is the most probable molecular speed (with $\kappa$ being Boltzmann's constant, $m$ the molecular mass, and $T$ the gas temperature). Thus $x$ and $y$ are the optical frequency shift and collision frequency $(\sim p / \eta)$ normalized with respect to $K a$, which is on the order of the frequency of a sound wave in the gas with wavelength $\Lambda_{\mathrm{s}}=2 \pi / K$. (Note that $a=(2 / \gamma)^{1 / 2}$ $c_{\mathrm{s}}$, where $c_{\mathrm{s}}$ is the speed of sound and $\gamma$ is the ratio of specific heats.) Alternatively, $y$ can be interpreted as the ratio of the acoustic wavelength involved in the scattering, $\Lambda_{\mathrm{s}}$, to the molecular mean free path.

For a single-component, low density gas, where $y<<1$, the normalized spectrum of the Rayleigh scattered light is given by the Gaussian

$$
S(x) d x=\frac{1}{\sqrt{\pi}} \exp \left[-\left(x-\frac{u_{K}}{a}\right)^{2}\right] d x
$$

The spectral width is proportional to the square root of the gas temperature. And the spectral peak is shifted by 
a frequency proportional to the component of the bulk velocity in the $\mathbf{K}$ direction (i.e., $u_{\mathrm{K}}=\mathbf{K} \cdot \mathbf{u} / \mathrm{K}$ ).

Note that in this limiting case for low density gases, the spectral shape is not a function of the $y$ parameter. However, for higher density gases (where $y$ $\sim 1$ ), the spectrum is no longer Gaussian and is a function of $y$. For $y \gg 1$ (high density gases), the scattering spectrum is strongly influenced by collective effects and is characterized by a central peak and two sidebands. The sidebands can be thought of as being caused by scattering from thermally excited random acoustic waves and is referred to as the BrillouinMandelstam doublet. A continuum theory ${ }^{12}$ can be used to model the spectrum here. The spectral shape in this regime is only a function of the $y$ parameter. However, the spectrum in the transition regime, where $y \sim 1$, requires a more detailed kinetic theory. We used the Tenti S6 model ${ }^{13}$ for this work. This model requires the shear viscosity, the thermal conductivity, the internal specific heat, and the bulk viscosity of the gas. In all cases, the Rayleigh scattering spectral shape is a function of the gas thermodynamic properties, which forms the basis for a diagnostic to measure gas density, temperature, and velocity.

Figure 1 shows typical spectra calculated using the Tenti S6 model. Note that for nitrogen at ambient conditions ( $p=1 \mathrm{~atm}, T=300 \mathrm{~K}$ ), using $514.5 \mathrm{~nm}$ laser light, and $90^{\circ}$ scattering, $y=0.77$, the simple Gaussian spectral model is not appropriate. For these calculations we used the shear viscosity and thermal conductivity provided by the FLUID program ${ }^{14}$, and the bulk viscosity value was obtained from the literature ${ }^{15}$.

In our work with air, we used air values for shear viscosity and thermal conductivity, the mean molecular weight of air, and the nitrogen values of internal specific heat and bulk viscosity. Although this is not strictly correct (since air should be treated as a mixture of gases), we believe that it does not result in significant errors.

The Rayleigh scattered light collected from one vantage point contains sufficient information to determine the gas density, temperature, and one component of the bulk velocity. (Spectra measured at other $\mathbf{K}$ vectors would give other velocity components.).

\section{Fabry-Perot interferometer}

The Fabry-Perot instrument function (transmission of a single frequency source) is ${ }^{16}$

$$
I_{F P}(\psi)=\left[1+F \sin ^{2}\left(\frac{\psi}{2}\right)\right]^{-1}
$$

where $\psi$ is the phase change (neglecting any phase change on reflection) of the light between successive reflections given by

$$
\psi\left(f, \theta_{r}\right)=\frac{4 \pi f \mu d \cos \theta_{r}}{c}
$$

Here, $\mu$ is the refractive index of the medium in the Fabry-Perot cavity, $d$ is the Fabry-Perot mirror spacing, $\theta_{r}$ is the angle between the ray and the optic axis, and $F$ $=1 /\left(\sin ^{2}\left(\pi / 2 N_{\mathrm{E}}\right)\right.$ where $N_{\mathrm{E}}$ is the effective finesse. The image of a monochromatic extended source located in the object plane consists of a series of unequally spaced concentric rings. With the innermost fringe located on the optical axis (i.e., having zero radius), the other fringe radii occur at $\theta_{r, n}=\left(n \lambda_{o} / d\right)^{1 / 2}$ for $n=1,2,3, \cdots$, Note that although the fringes are unequally spaced, their frequency separations are all equal to the free spectral range $F S R=c / 2 d$.

The phase $\psi$ in the Fabry-Perot instrument function can be written as a first order approximation in terms of the non-dimensional frequency $x$ (defined in the previous section) as

$$
\psi(x)=a_{1}+a_{2} x
$$

where the first term represents the contribution due to the change in phase (relative to the phase along the optical axis) resulting from the optical path length in the interferometer at angle $\theta_{\mathrm{r}}$, and the second term is due to the change in the frequency. These terms can be written

$$
a_{1}=\psi_{o p}-\psi_{o} \frac{\theta_{r}^{2}}{2}, \quad a_{2}=\psi_{o} \frac{\lambda}{\Lambda_{s}} \frac{a}{c}
$$

with

$$
\psi_{o}=\frac{4 \pi d_{o}}{\lambda_{o}} \quad, \quad \psi_{o p}=\bmod \left[\frac{4 \pi d}{\lambda}, 2 \pi\right]
$$

Because of the first order approximation, $d_{\mathrm{o}}$ need only be the characteristic mirror spacing (i.e., it does not need to be the actual, high-precision value of the spacing $d$ used in the expression for $\psi_{\mathrm{op}}$ ).

An example of a typical Fabry-Perot instrument function is shown in figure 2. The transmission of a single frequency source is plotted over several orders as a function of $x$. The parameters used are those of the experiment described below.

Measurement of spectrum with Fabry-Perot

Light passed through the Fabry-Perot interferometer is filtered by the interferometer instrument function. If the light is detected with an 
array detector, we can write the number of detected photons for the $q^{\text {th }}$ pixel as

$$
\begin{aligned}
<N_{D q}> & =\frac{1}{\Delta A} \iint_{\Omega} \int_{\Delta A-\infty}^{\infty}\left[A_{R} S_{R}\left(x-\frac{u_{K}}{a}, \theta_{r}\right)\right. \\
& \left.+A_{W} \delta(x)\right] I_{F P}\left(x, \theta_{r}\right) \mathrm{d} x \mathrm{~d} A d \Omega+B_{q}
\end{aligned}
$$

where $\left\langle N_{\mathrm{Dq}}\right\rangle$ is the number of detected photons for the $q_{\text {th }}$ pixel, $\Delta A$ is the pixel area, $A_{\mathrm{R}}$ is the amplitude of Rayleigh scattered light, $A_{\mathrm{W}}$ is the amplitude of the stray scattered laser light, and $B_{\mathrm{q}}$ represents broadband background light. Examples of typical Fabry-Perot images are shown in figure 3. Only the innermost fringe is displayed, which corresponds to the experiment described below. Figure $3 \mathrm{a}$ is the image for single frequency laser light, and figure $3 b$ is the image for Rayleigh scattered light.

For the general case, the three integrations shown in equation 10 must be carried out. However, we may simplify this by making the following assumptions. First, the integration over the solid angle $\Omega$ may be replaced by the angles defined by the incident and scattered beams. This, in effect, assumes that broadening of the scattered spectrum is negligible. For the work presented here, the convergence angle of the incident beam and the solid collection angle are such that $\mathbf{K}$ vector broadening is negligible.

The second simplification we make is in the integration over each pixel. We replace this integral with a single value obtained by evaluating the expression at the center of the pixel. Alternatively, we can use a numerical integration over the pixel which uses nine points within the pixel. Since the results using these two schemes show only minor differences, we use the single point scheme to reduce computer processing time.

\section{Lower bounds for measurement uncertainty}

Since Rayleigh scattering is a relatively weak process, the uncertainty in the measurements often is set by the photon statistical noise (shot noise), which determines the lower bound on measurement uncertainty. For example, the variance in the number of photoelectron counts for a Poisson process is equal to the mean number of counts. Thus the lower bound for the relative uncertainty in the measurement of gas density $\rho$ is equal to the square root of the reciprocal of the mean counts.

In general, estimates of the measurement uncertainty for the technique can be obtained by calculating the Cramer-Rao lower bound ${ }^{17}$. The variance of the estimate of a parameter $\alpha_{i}$ (e.g., temperature or velocity, etc.) is given by

$$
V\left(\alpha_{i}\right)=\left[\Gamma^{-1}\right]_{i i}
$$

where $\Gamma$ is the Fisher information matrix with elements

$$
\Gamma_{i j}=\sum_{q} \frac{1}{\left\langle N_{D_{q}}\right\rangle} \frac{\partial\left\langle N_{D_{q}}\right\rangle}{\partial \alpha_{i}} \frac{\partial\left\langle N_{D_{q}}\right\rangle}{\partial \alpha_{j}}
$$

For a Gaussian spectrum $(y<<1)$, the lower bounds for density, temperature and velocity uncertainties are

$\frac{\sigma(\rho)}{\rho}=\left(\frac{1}{N_{R}}\right)^{1 / 2}, \quad \frac{\sigma(T)}{T}=\left(\frac{2}{N_{R}}\right)^{1 / 2}, \quad \frac{\sigma\left(u_{K}\right)}{u_{K}}=\left(\frac{2}{\gamma}\right)^{1 / 2} \frac{1}{M_{K}\left(2 N_{R}\right)^{1 / 2}}$

where $N_{\mathrm{R}}$ is the number of detected photons and $u_{\mathrm{K}}$ is the velocity component along the vector $\mathbf{K}$ (with $M_{\mathrm{K}}$ being the corresponding Mach number).

\section{Example:}

We consider an example using parameters similar to the experiment discussed later. For air at $300 \mathrm{~K}$ and 14.7 psia, with $200 \mathrm{~mW}$ of $514.5 \mathrm{~nm}$ laser light and a 1 sec exposure, a probe volume length $L_{\mathrm{x}}=1.5 \mathrm{~mm}, \mathrm{f} / 6$ collection optics, and collection efficiency $\varepsilon=10 \%$, the number of detected photons (from eq. 1) $N_{\mathrm{R}}$ is $2.9 \times 10^{6}$. Thus the lower bound on the measurements, using an ideal instrument, of density, temperature, and velocity are $\sigma(\rho) / \rho=0.059 \%, \sigma(T)=0.13 \mathrm{~K}$, and $\sigma\left(u_{\mathrm{K}}\right)=0.17$ $\mathrm{m} / \mathrm{sec}$. Of course, this cannot be achieved in practice because it only includes the uncertainty due to photon statistical noise. Making the measurement with any real instrument will result in larger uncertainties. However, it does show the potential for very accurate measurements based on molecular Rayleigh scattering.

We can also calculate the lower bounds for measurement uncertainties using a model for the actual instrument used in our work (Fabry-Perot interferometer and CCD array detector). We assume that the collected light is distributed over a 95 pixel diameter (about 7000 pixels) on the CCD After the filtering of the light by the Fabry-Perot is taken into account, about 400 photons (maxium) are detected by each pixel. In addition to the photon shot noise, we include CCD read noise of 2 photoelectrons rms. Using the model function given by equation 10 for three parameters $\alpha_{i}$ (amplitude, temperature, and velocity) with zero background and zero stray laser light, the lower bounds given by equation 11 are shown as a function of interferometer finesse on figure 4 . This shows that the optimum finesse is about 10 . (The reason that the 
optimum finesse occurs at a relatively low finesse is that higher resolutions decrease the number of photons transmitted through the interferometer, which causes in increase in the photon statistical noise). At the optimum finesse, the uncertainty for a temperature measurement is about a factor of four larger than the uncertainty found above for an ideal instrument. And, the uncertainty for a velocity measurement is about a factor of six larger that than of an ideal instrument.

Other potential error sources that can broaden the spectrum, thus affecting the temperature determination, include aperture broadening due to the range of $\mathbf{K}$ vectors across the receiving optics aperture and broadening caused by turbulence ${ }^{4}$. In addition to these fundamental error sources, increased measurement uncertainty results from various environmental effects such as temperature variations, high acoustic noise levels, and vibration. In our measurements, it also appears that speckle may contribute to the overall noise seen in the Rayleigh scattering images.

\section{$\underline{\text { Experiment }}$}

\section{Apparatus}

The optical setup used for this study is shown in figure 5. The beam from an argon-ion laser $(514 \mathrm{~nm}$, $0.5 \mathrm{~W}$ ) is focused by lens L1 (focal length $100 \mathrm{~mm}$ ) into a multimode $200 \mu \mathrm{m}$ core diameter, $20 \mathrm{~m}$ long optical fiber. This fiber is routed from the room containing the laser to another room where the experiment is located. The laser light exiting the fiber is collimated by lens L2 (focal length $160 \mathrm{~mm}$ ). The collimated beam is passed through a polarizing beam splitter PBS which only transmits light that is linearly polarized with electric vector normal to the plane of optical table. The light reflected upward by the beam splitter is directed into a light trap (not shown on figure). This light would not contribute to the Rayleigh scattering signal since the plane of the Rayleigh scattering is in the plane of the table. It could, however, contribute to detected stray light if it is depolarized when scattered from surfaces near the probe volume. The beam transmitted by the beam splitter is focused by lens L3 (focal length 200 $\mathrm{mm})$. The beam diameter at the probe volume is thus about $0.25 \mathrm{~mm}$. The measured laser power delivered to the probe volume was $200 \mathrm{~mW}$. Rayleigh scattered light is collected by lens L4 (f/5.5, focal length 250 $\mathrm{mm}$ ) and focused by lens L5 (focal length $160 \mathrm{~mm}$ ) into a $1.0 \mathrm{~mm}$ core diameter, $20 \mathrm{~m}$ long, optical fiber. The length of the probe volume is thus about $1.5 \mathrm{~mm}$ (defined by the image of the $1 \mathrm{~mm}$ fiber at the measurement region). The $1 \mathrm{~mm}$ fiber is routed back to the room containing the laser and detection system.
Light from the output of the fiber is collimated by lens L7 (focal length $100 \mathrm{~mm}$ ) and passed through a planar mirror Fabry-Perot interferometer $(70 \mathrm{~mm}$ mirror diameter, $15.24 \mathrm{~mm}$ mirror spacing $[9.84 \mathrm{GHz}$ free spectral range]) operated in the static, imaging mode, and focused by lens L8 (135 mm, $35 \mathrm{~mm}$ camera lens) onto a liquid nitrogen cooled CCD camera (1732 x 532, $15 \mu \mathrm{m}$ square pixels). The lens L2 (100 mm focal length) and lens L3 (135 $\mathrm{mm}$ focal length) were selected so that the light emitted from the optical fiber forms a $1.35 \mathrm{~mm}$ (90 pixel) diameter image.

Additional optics were included to provide for a reference image consisting of light at the unshifted laser frequency. To accomplish this, several components could be placed in the optical path. Mirror M reflected light from the $200 \mu \mathrm{m}$ fiber, which was focused by lens L6 onto a diffuser DIF located in front of the $1.0 \mathrm{~mm}$ fiber. Diffusely scattered laser light then entered the fiber. A neutral density filter NDF could also be placed in this light path to reduce the intensity of the reference light. The mirror M, diffuser DIF, and neutral density filter were mounted on remotely controlled pneumatic linear actuators. Finally, a prism assembly could be placed in the light path between the Fabry-Perot interferometer Fabry-Perot interferometer and the CCD camera. This served to direct light into a standard video camera. The video signal from this camera was digitized by a frame grabber card in a 486 PC. A computer program analyzed this image and generated signals to control the Fabry-Perot mirror alignment. The prism assembly, which was located in the room with the operator, was manually positioned. The other components, which were located in the room with the experiment, were remotely controlled by the operator.

The procedure for taking data was as follows. The prism assembly, mirror and diffuser were placed in the beam path. The automatic Fabry-Perot interferometer alignment system was operated to maximize the finesse and to lock the reference fringe at a specified diameter. Most of the data was taken with a reference fringe diameter of 45 pixels. When the operator was ready to take data, the prism assembly was removed from the beam path and the neutral density filter was placed in the beam path. The reference light (fig. 3a) was then imaged by the CCD camera. This image was stored on a 686 PC. The mirror $\mathrm{M}$ and diffuser DIF were then removed from the beam path, allowing the Rayleigh scattered light from the probe volume to be imaged through the Fabry-Perot onto the CCD camera (fig. 3b). This image was also stored for later analysis. Only a 200 pixel square region of the CCD was used for these images to reduce the computer storage requirements. 
Two experiments were conducted. The first used a subsonic air jet (shown on figure 5). The nozzle exit diameter was $8.7 \mathrm{~mm}$. A $0.2 \mu \mathrm{m}$ filter was used in the air supply to eliminate particulates. The pressure in the nozzle plenum was measured with an electronic pressure gauge, and the plenum temperature was measured with a copper-constantan thermocouple. Isentropic flow relations were then used to calculate the jet temperature and velocity.

The second experiment used a low-speed electrically heated air flow (11 mm dia.) surrounded by a co-flow (52 $\mathrm{mm}$ dia.) of room temperature air. Filters $(0.2 \mu \mathrm{m})$ were used for both the heated air and the coflow air. All reported measurements were taken $9 \mathrm{~mm}$ from the exit plane of the heated jet. The heated jet was located above the probe volume with the flow directed downward (not shown on figure 5).. The velocity component seen by the Rayleigh scattering system was thus essentially zero. The temperature at the probe volume was measured with a 0.010 inch diameter copper-constantan thermocouple.

\section{Data processing}

The reference image (fig. 3a) was first analyzed using a weighted, nonlinear least square fitting routine. After the average dark image (obtained from 10 images) was subtracted from the reference image, a circular region of 95 pixel diameter was analyzed using a five parameter fit to a model function based on the ideal Fabry-Perot instrument function (eq. 5). The parameters were the amplitude, the finesse, the fringe radius (interferometer phase), the coordinates of the center of the fringe, and the uniform background level. The noise was modeled using a ratio of photoelectronsto-CCD counts of 4.5 and an rms read noise of 2 photoelectrons. The photoelectrons were assumed to have Poisson statistics.

The Rayleigh scattering images were then processed using a five parameter fit to a model function (eq. 10) based on the S6 Tenti spectrum ${ }^{13}$ over a 95 pixel diameter region. The interferometer finesse, fringe center, and fringe diameter obtained from the reference image fit were used. The fitting parameters were the Rayleigh scattering amplitude, velocity, temperature, stray laser light amplitude, and uniform background. The total processing time on a $166 \mathrm{MHz}$ 586 PC was about two minutes for each image. However, no attempt was made to optimize the software to reduce the processing time.

\section{$\underline{\text { Results }}$}

Velocity data obtained from the subsonic free air jet are shown in figure 6 . One second exposures were used for both the reference and Rayleigh scattering images. Reading numbers 1 to 8 and 17 to 21 were for zero velocity. The other data were for velocities in the range of 200 to $300 \mathrm{~m} / \mathrm{sec}$. The reference fringe diameter was set at 40 pixels for readings 1 to 28,45 pixels for readings 29 to 32 , and 50 pixels for readings 33 to 36 . The probe volume was located $14.5 \mathrm{~mm}$ from the nozzle exit for readings 1 to 16 , and $16.5 \mathrm{~mm}$ for readings 17 to 36 . The velocity calculated from the measured total temperature and total pressure are shown. The velocities obtained from the Rayleigh scattering measurements show very good agreement with the isentropic velocities, with the mean deviation from the isentropic velocities for the 36 measurements being $-0.4 \mathrm{~m} / \mathrm{sec}$.

The temperatures obtained from the subsonic free jet images along the temperatures from the isentropic flow relations are shown in figure 7. Note that the agreement is not good. The Rayleigh scattering temperatures are biased high by about $30 \mathrm{~K}$.

Velocity data for 1 and 10 second exposures for the low speed heated jet are shown in figures 9 and 11 . The mean deviation from the true velocity (essentially zero for these measurements) is about $-5.4 \mathrm{~m} / \mathrm{sec}$ for the 1 second exposure data (fig. 9) and about $-3.9 \mathrm{~m} / \mathrm{sec}$ for the 10 second exposure data (fig. 11).

Temperatures obtained from the heated low-speed jet are shown in figures 8 and 10, along with the temperatures obtained from the thermocouple measurements. These do not show good agreement. The temperatures obtained from the Rayleigh scattering images are biased high with respect to the thermocouple data by 10 to $15 \%$. The explanation for this is not completely understood. Note that temperature measurements are sensitive to the shape of both the Fabry-Perot instrument function and the Rayleigh scattering spectrum. On the other hand, velocity is not particularly sensitive to the spectral shape since only the location of the spectral peak is needed. There are several possibilities for the errors in the temperature. One is that the model for either the Fabry-Perot instrument function or the Rayleigh scattering spectrum is not realistic. For example, a uniform background is assumed in the reduction of the Rayleigh scattering images. The temperature obtained is a very sensitive function of the value of the background level. Figure 12 shows four parameter fits obtained with the background fixed. A small deviation in the background can cause large changes in the temperature. This may be caused by the region over which the fit is done being only a fraction of the free spectral range. Better results may be obtained by using a more accurate model, or by using a larger fitting region that encompasses a greater part of the free spectral range. 


\section{Concluding remarks}

The application of molecular Rayleigh scattering for velocity and temperature measurements of gas flows was implemented using a planar mirror Fabry-Perot interferometer operated in the imaging mode with a cooled CCD camera. Light from an argon-ion laser was transmitted via an optical fiber to a remote location where the experiment was set up. The Rayleigh scattered light was transmitted through another optical fiber to the room where the Fabry-Perot and CCD camera were located. This arrangement demonstrated the capability of placing sensitive equipment in a controlled environment, while making measurements in harsh environments.

Two flows were examined. The first was a subsonic free air jet. Velocity measurements obtained with the Rayleigh scattering system showed very good agreement with velocities calculated from isentropic flow relations. On other hand, the temperature measurements did not show equally good agreement. The second flow studied was a low-speed, heated air flow. Here the velocity was essentially zero. Again, the accuracy of the velocity measured with the Rayleigh scattering system was good, with the velocity measurements for both sets of measurements being within $5 \mathrm{~m} / \mathrm{sec}$ of the actual values. The Rayleigh scattering temperature measurements, however, showed systematic errors on the order of $10-15 \%$. It is felt that the model used for the data reduction did not accurately represent the overall distribution of the collected light. It should be pointed out that no adjustable parameters were used in the data reduction. Only measured (e.g, Fabry-Perot interferometer mirror spacing) and known physical parameters (e.g., air properties) were used.

In summary, the Rayleigh scattering measurement system appears capable of making accurate velocity measurements. However, the technique used here for temperature measurements requires additional development.

\section{References}

${ }^{1}$ Seasholtz, R.G., "2D Velocity and Temperature Measurements in High Speed Flows based on Spectrally Resolved Rayleigh Scattering", NATO Advanced Research Workshop, New Trends in Instrumentation for Hypersonic Research, ONERA Le Mauzac, France, April 27 - May 1, 1992.

${ }^{2}$ Lock, J.A., Seasholtz, R.G., and John, W.T., "RayleighBrillouin Scattering to Determine One-Dimensional Temperature and Number Density Profiles of a Gas Flow Jet", Appl. Opt., Vol. 31, no. 15, 1992, pp. 28392848.

${ }^{3}$ Seasholtz, R.G., "Single-Shot Spectrally Resolved UV Rayleigh Scattering Measurements in High Speed
Flow", Eighth International Symposium of Applications of Laser Techniques to Fluid Mechanics, Lisbon, July 8-11, 1996. (Also NASA TM-107323).

${ }^{4}$ Seasholtz, R.G., Zupanc, F.J., and Schneider, S.J., "Spectrally Resolved Rayleigh Scattering Diagnostic for Hydrogen-Oxygen Rocket Plume Studies", $J$. Propulsion and Power, Vol. 8, No.5, 1992, pp. 935942.

${ }^{5}$ Seasholtz, R.G., Buggele, A.E., and Reeder, M.F., "Instantaneous Flow Measurements in a Supersonic Wind Tunnel Using Spectrally Resolved Rayleigh Scattering”, SPIE International Symposium on Optical Science, Engineering, and Instrumentation, San Diego, July 9-14, 1995.

${ }^{6}$ Kourous, H.E., and Seasholtz, R.G., "Fabry-Perot Interferometer Measurement of Static Temperature and Velocity for ASTOVL Model Tests", FED-Vol.1, Laser Anemometry - 1994: Advances and Applications, ASME, 1994.

${ }^{7}$ Anderson, D.G., et al., "Fiber-optic-bundle delivery system for high peak power laser particle image velocimetry illumination", Rev. Sci., Instrum., Vol. 67, No. 8, 1996, pp. 2675-2679.

${ }^{8}$ Young, A.T., "Rayleigh Scattering", Physics Today, January 1982, pp. 42-48.

${ }^{9}$ Buckingham, A.D., and Graham, C., "The Density Dependence of the Refractivity of Gases", Proc. R. Soc. Lond. Vol. A337, 1974, pp. 275-291.

${ }^{10}$ Watson, H.E., and Ramaswamy, K.L., "The Refractive Index Dispersion and Polarization of Gases", Proc. $R$. Soc. Lond., Vol. A156 (1936), pp. 144-157.

${ }^{11}$ Gardiner, W.C. Jr., Hidaka, Y. \& Tanzawa, T., "Refractivity of Combustion Gases", Comb. and Flame, Vol. 40, 1981, pp. 213-219.

${ }^{12}$ Clark, N.A., "Inelastic Light Scattering from Density Fluctuations in Dilute Gases. The Kinetic Hydrodynamic Transition in Monatomic Gas", Phys. Rev. Vol. A12, 1975, pp. 232-244.

${ }^{13}$ Tenti, G., Boley, C.D. and Desai,R.C., "On the Kinetic Model Description of Rayleigh Brillouin Scattering from Molecular Gases", Can. J. Phys. Vol 53, 1974, pp. 285-290.

${ }^{14}$ Fessler, T.E., FLUID: “A Numerical Interpolation Procedure for Obtaining Thermodynamic and Transport Properties of Fluids", NASA TM X-3572, 1977.

${ }^{15}$ Boley, C.D. Desai, R.C. \& Tenti, G., "Kinetic Models and Brillouin Scattering in a Molecular Gas", Can. J. Phys. Vol. 50, 1972, pp. 2158-2173.

${ }^{16}$ Vaughan, J.M., The Fabry Perot Interferometer, History, Theory, Practice and Applications, Adam Hilger, Bristol, 1989, pp. 89-112.

${ }^{17}$ Whalen, A.D., Detection of Signals in Noise, Academic Press, New York, 1971, pp. 324-231. 


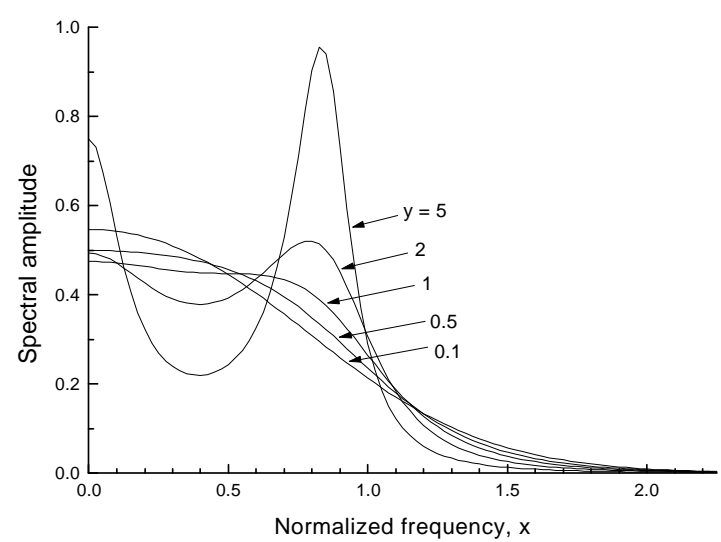

Fig. 1 Rayleigh scattering spectra calculated using Tenti S6 model for several values of the $y$ parameter. The ratio of shear viscosity to bulk viscosity used was 1.407 , the internal specific heat was 1.0 , and the ratio of shear viscosity to thermal conductivity was 0.2 .

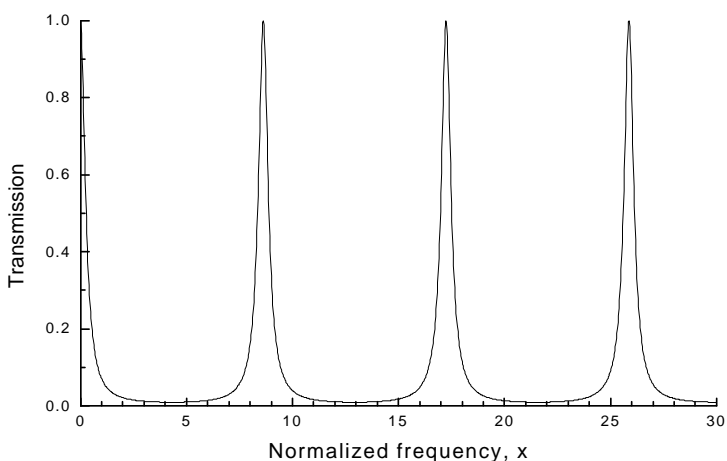

Fig. 2 Fabry-Perot interferometer instrument function for free spectral range $9.84 \mathrm{GHz}$, finesse $=16, K a=$ $7.168 \times 10^{9}$.

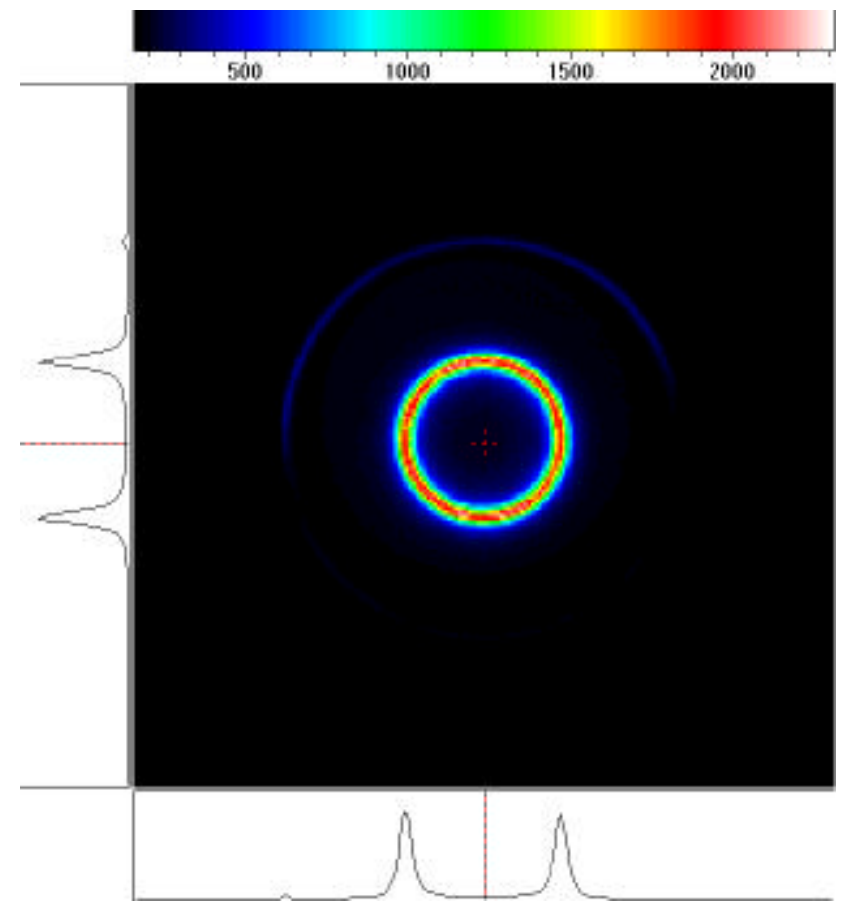

(a)

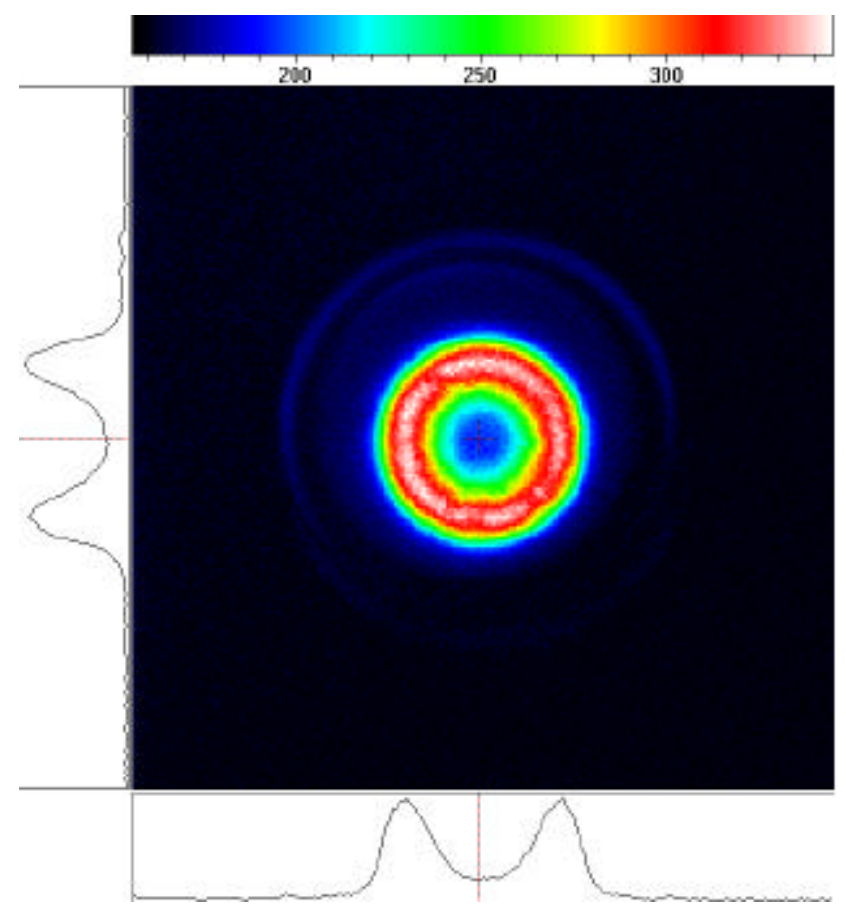

(b)

Fig. 3 (a) Image of Fabry-Perot instrument function; (b) Rayleigh scattering image for $T=297 \mathrm{~K}$; exposures are $10 \mathrm{sec}$; a single fringe is shown along with horizontal and vertical plots of a single row and a single column of pixels through the center of the fringe patterns. 


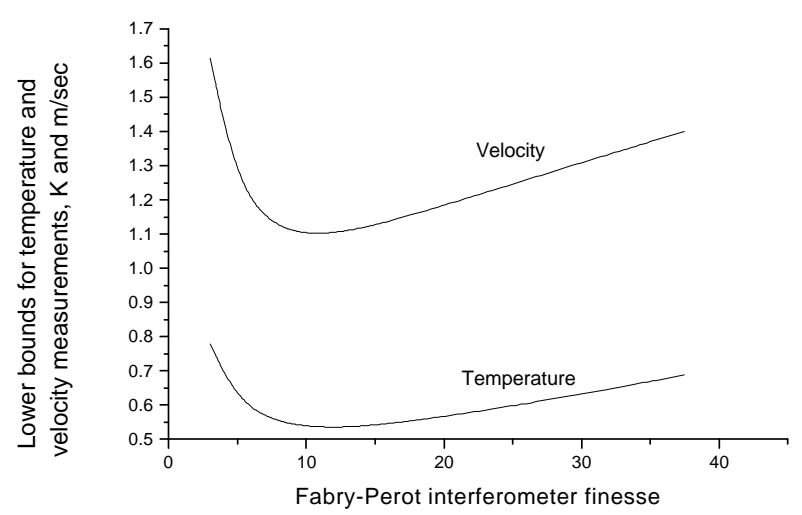

Fig. 4 Calculated lower bounds for temperature and velocity as function of Fabry-Perot interferometer finesse; number photoelectrons $/$ pixel $=400$.

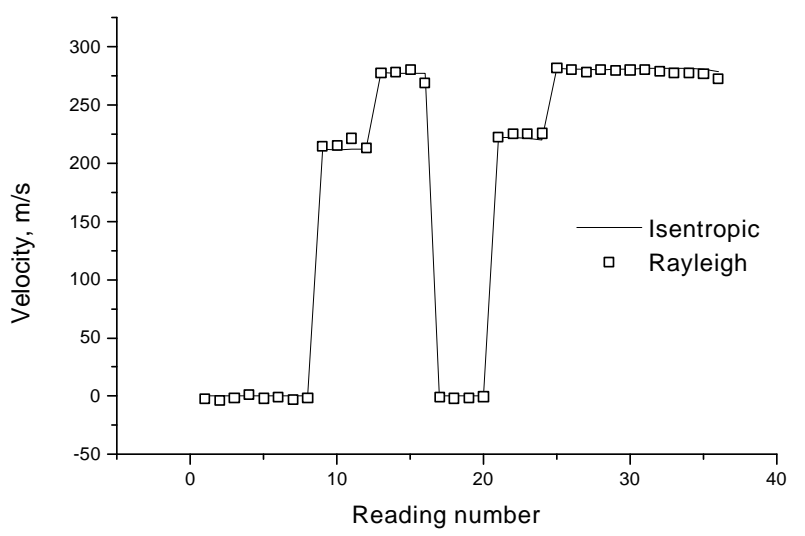

Fig. 6 Velocity of free jet measured with Rayleigh scattering system and calculated from isentropic flow equations.

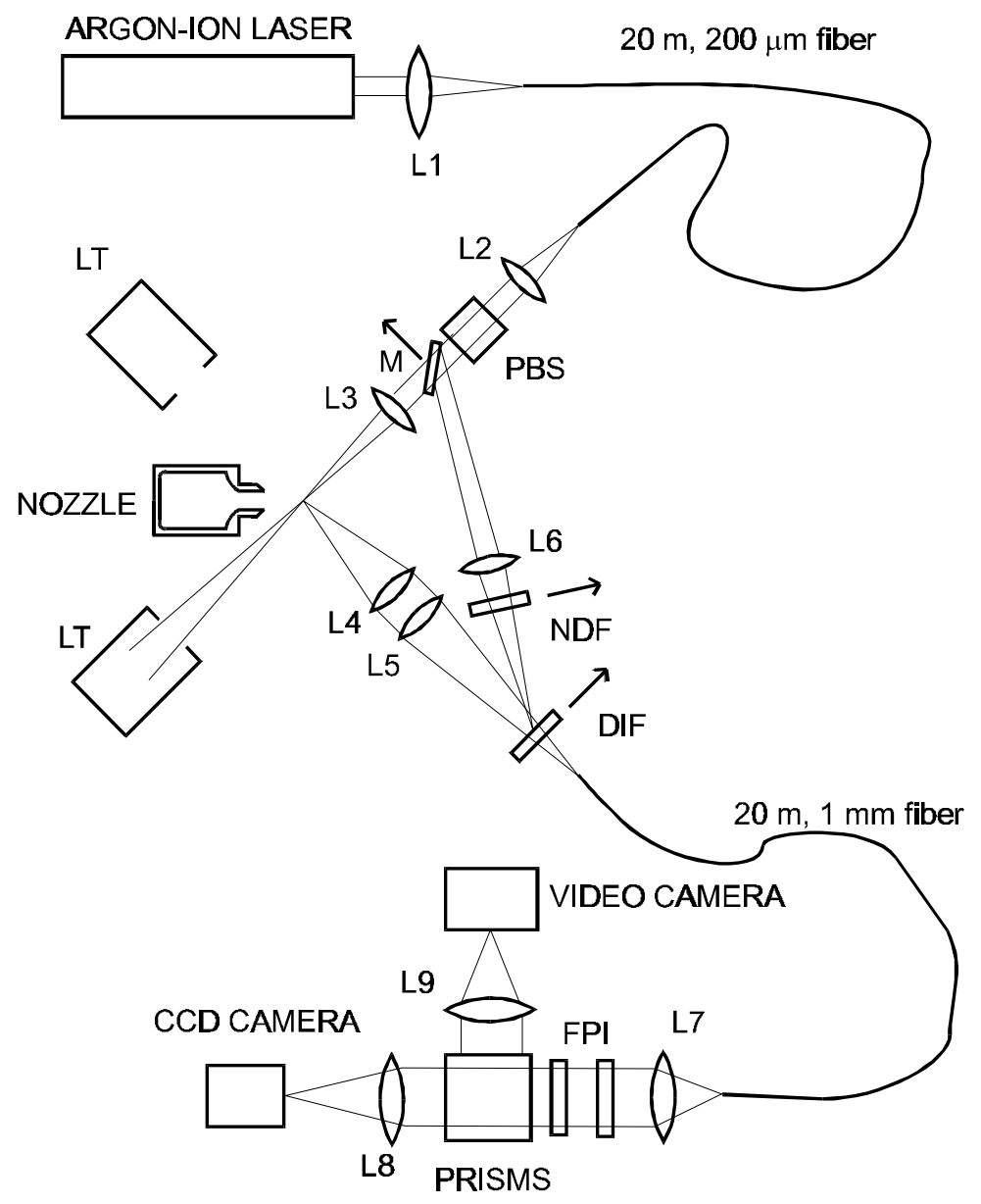

Fig. 5 Optical layout of Rayleigh scattering experiment. 


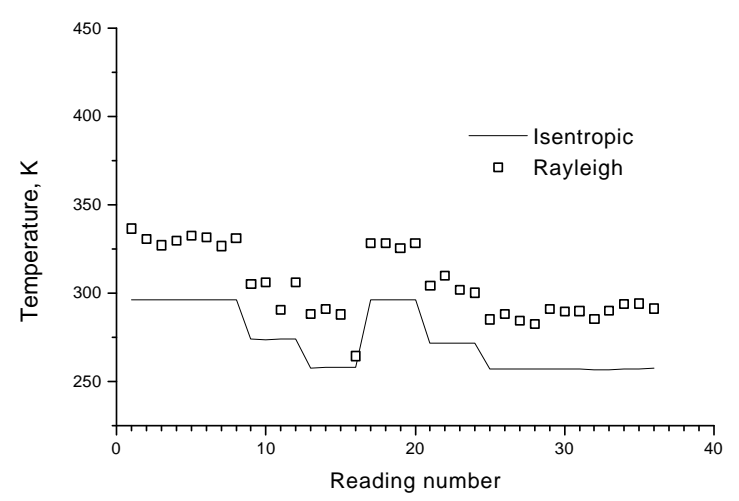

Fig. 7 Temperature of free jet measured with Rayleigh scattering system and calculated from isentropic flow equations.

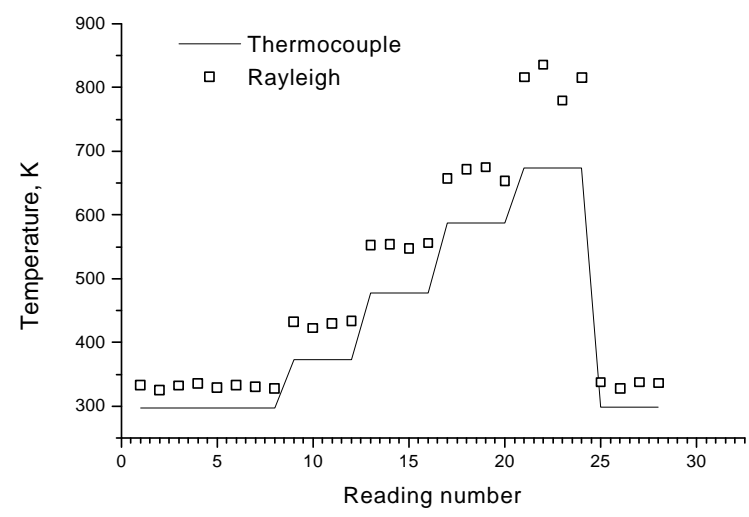

Fig.8 Temperature of low speed heated jet measured with Rayleigh scattering and thermocouple (1 sec exposure).

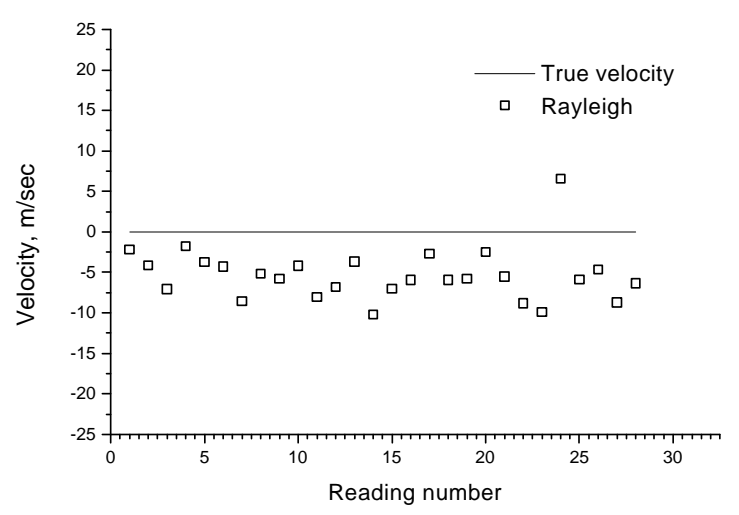

Fig. 9 Velocity of low speed heated jet measured with Rayleigh scattering (1 sec exposure) with zero velocity shown as reference.

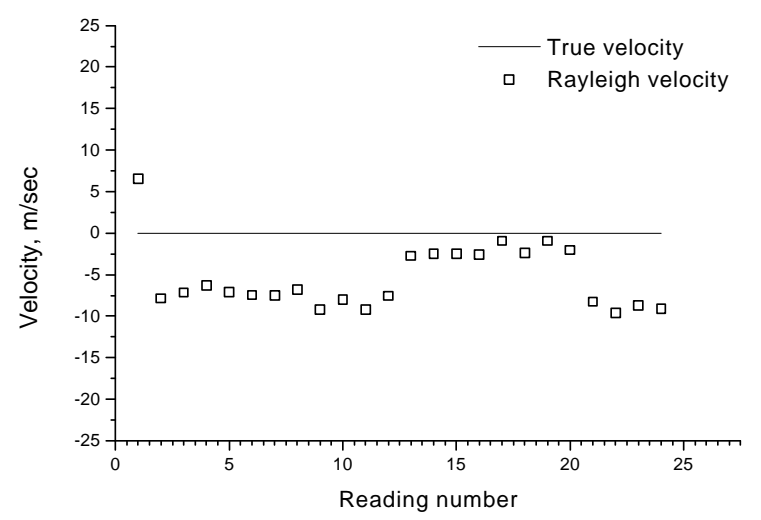

Fig. 10 Temperature of low speed heated jet measured with Rayleigh scattering and thermocouple (10 sec exposure).

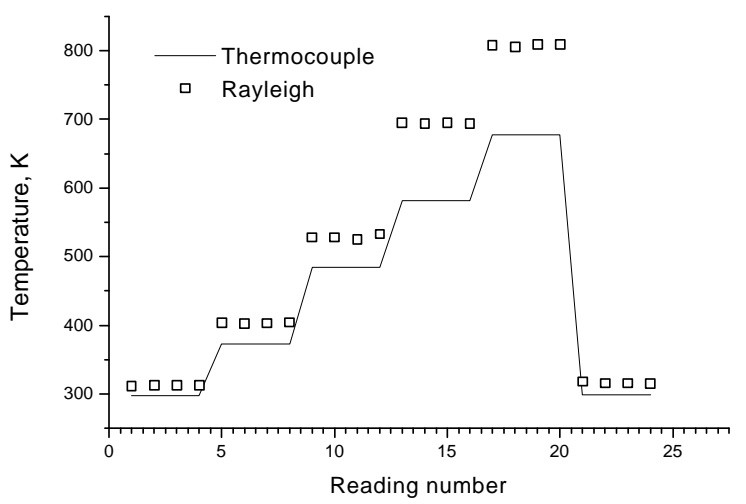

Fig. 11 Velocity of low speed heated jet measured with Rayleigh scattering (10 sec exposure) with zero velocity shown as reference.

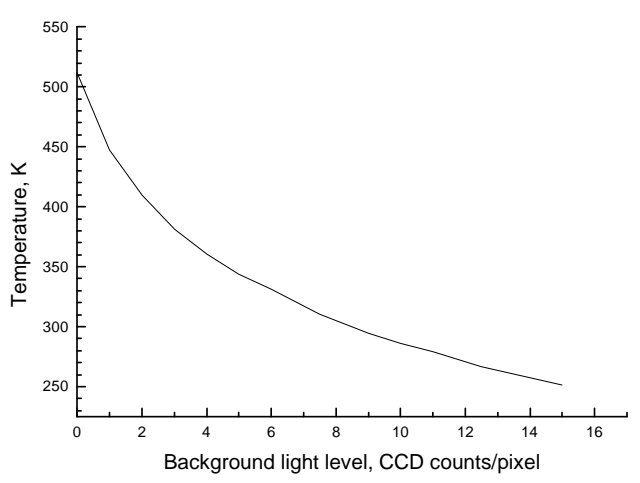

Fig. 12 Effect of background level (fixed) on temperature found from least-squares fit to Rayleigh scattering Fabry-Perot interferometer image. 
Public reporting burden for this collection of information is estimated to average 1 hour per response, including the time for reviewing instructions, searching existing data sources, gathering and maintaining the data needed, and completing and reviewing the collection of information. Send comments regarding this burden estimate or any other aspect of this collection of information, including suggestions for reducing this burden, to Washington Headquarters Services, Directorate for Information Operations and Reports, 1215 Jefferson Davis Highway, Suite 1204, Arlington, VA 22202-4302, and to the Office of Management and Budget, Paperwork Reduction Project (0704-0188), Washington, DC 20503.

\begin{tabular}{|l|l|r|}
\hline 1. AGENCY USE ONLY (Leave blank) & $\begin{array}{c}\text { 2. REPORT DATE } \\
\text { April } 1998\end{array}$ & $\begin{array}{r}\text { 3. REPORT TYPE AND DATES COVERED } \\
\text { Technical Memorandur }\end{array}$
\end{tabular}

\section{TITLE AND SUBTITLE}

5. FUNDING NUMBERS

Rayleigh Scattering Diagnostic for Measurement of Temperature and Velocity in Harsh Environments

6. AUTHOR(S)

Richard G. Seasholtz and Lawrence C. Greer III

7. PERFORMING ORGANIZATION NAME(S) AND ADDRESS(ES)

National Aeronautics and Space Administration

Lewis Research Center

Cleveland, Ohio 44135-3191

WU-519-20-53-00

National Aeronautics and Space Administration

Washington, DC 20546-0001

8. PERFORMING ORGANIZATION REPORT NUMBER

E-11137

\section{SPONSORING/MONITORING AGENCY NAME(S) AND ADDRESS(ES)}

10. SPONSORING/MONITORING AGENCY REPORT NUMBER

NASA TM-1998-206980

AIAA-98-0206

\section{SUPPLEMENTARY NOTES}

Prepared for the 36th Aerospace Sciences Meeting \& Exhibit sponsored by the American Institute of Aeronautics and Astronautics, Reno, Nevada, January 12-15, 1998. Responsible person, Richard G. Seasholtz, organization code 5520, (216) 433-3754.

12a. DISTRIBUTION/AVAILABILITY STATEMENT 12b. DISTRIBUTION CODE

Unclassified - Unlimited

Subject Category: 35

Distribution: Nonstandard

This publication is available from the NASA Center for AeroSpace Information, (301) 621-0390.

13. ABSTRACT (Maximum 200 words)

A molecular Rayleigh scattering system for temperature and velocity measurements in unseeded flows is described. The system is capable of making measurements in the harsh environments commonly found in aerospace test facilities, which may have high acoustic sound levels, varying temperatures, and high vibration levels. Light from an argon-ion laser is transmitted via an optical fiber to a remote location where two flow experiments were located. One was a subsonic free air jet; the second was a low-speed heated air jet. Rayleigh scattered light from the probe volume was transmitted through another optical fiber from the remote location to a controlled environment where a Fabry-Perot interferometer and cooled CCD camera were used to analyze the Rayleigh scattered light. Good agreement between the measured velocity and the velocity calculated from isentropic flow relations was demonstrated $(<5 \mathrm{~m} / \mathrm{sec})$. The temperature measurements, however, exhibited systematic errors on the order of $10-15 \%$.

\section{SUBJECT TERMS}

Rayleigh scattering; Fabry-Perot interferometers

16

A03

\begin{tabular}{|c|c|}
\hline $\begin{array}{c}\text { 17. SECURITY CLASSIFICATION } \\
\text { OF REPORT } \\
\text { Unclassified }\end{array}$ & $\begin{array}{c}\text { 18. SECURITY CLASSIFICATION } \\
\text { OF THIS PAGE } \\
\text { Unclassified }\end{array}$ \\
\hline
\end{tabular}

NSN 7540-01-280-5500

19. SECURITY CLASSIFICATION
OF ABSTRACT
Unclassified

Standard Form 298 (Rev. 2-89)

Prescribed by ANSI Std. Z39-18 298-102 